\title{
Product Dimension of Forests and Bounded Treewidth Graphs
}

\author{
L. Sunil Chandran ${ }^{1}$ Rogers Mathew ${ }^{* 2}$ Deepak Rajendraprasad ${ }^{\dagger 1}$ Roohani Sharma ${ }^{1}$ \\ ${ }^{1}$ Department of Computer Science and Automation \\ Indian Institute of Science \\ Bangalore, India, 560012 \\ \{sunil, deepakr\}@csa.iisc.ernet.in, \\ roohani.sharma90@gmail.com \\ 2 Department of Mathematics and Statistics \\ Dalhousie University, Halifax, Canada - B3H 3J5 \\ rogersm@mathstat.dal.ca
}

\begin{abstract}
Submitted: Sep 10, 2012; Accepted: Sep 6, 2013; Published: Sep 20, 2013
Mathematics Subject Classifications: 05C05, 05C62
\end{abstract}

\begin{abstract}
The product dimension of a graph $G$ is defined as the minimum natural number $l$ such that $G$ is an induced subgraph of a direct product of $l$ complete graphs. In this paper we study the product dimension of forests, bounded treewidth graphs and $k$-degenerate graphs. We show that every forest on $n$ vertices has product dimension at most $1.441 \log n+3$. This improves the best known upper bound of $3 \log n$ for the same due to Poljak and Pultr. The technique used in arriving at the above bound is extended and combined with a well-known result on the existence of orthogonal Latin squares to show that every graph on $n$ vertices with treewidth at most $t$ has product dimension at most $(t+2)(\log n+1)$. We also show that every $k$-degenerate graph on $n$ vertices has product dimension at most $\lceil 5.545 k \log n\rceil+1$. This improves the upper bound of $32 k \log n$ for the same by Eaton and Rödl.
\end{abstract}

Keywords: product dimension, representation number, forest, bounded treewidth graph, $k$-degenerate graph, orthogonal Latin squares.

*Supported by an AARMS Postdoctoral Fellowship

${ }^{\dagger}$ Supported by Microsoft Research India PhD Fellowship 


\section{Introduction}

For a graph $G(V, E)$ and an $l \in \mathbb{N}$, a function $\phi_{G}: V \rightarrow \mathbb{N}^{l}$ is called an $l$-encoding of $G$ if

1. $\phi_{G}$ is an injection, and

2. $\forall u, v \in V,\{u, v\} \in E$ iff $\phi_{G}(u)$ and $\phi_{G}(v)$ differ in all $l$ coordinates.

The minimum $l$ such that an $l$-encoding of $G$ exists is called the product dimension of $G$ and is denoted by $\operatorname{pdim}(G)$. Some authors refer to it as the Prague dimension [10].

The product dimension of a graph $G$ was first defined in [15] by Nešetřil and Rödl as the minimum $l$ such that $G$ is an induced subgraph of a direct product (see Section 1.2) of $l$ complete graphs. It is easy to see that the two definitions of product dimension are equivalent. Another equivalent definition of the product dimension of a graph is as the minimum number of proper colorings of $G$ such that any pair of non-adjacent vertices get the same color in at least one of the colorings and not in all of them.

The concept of product dimension of a graph was first used to prove the GalvinRamsey property of the class of all finite graphs [15]. Thereafter, this area was separately explored by various people. In 1980, Lovász, Nešetřil and Pultr showed that the product dimension of a path on $n+1$ vertices (length $n$ ) is $\lceil\log n\rceil$ [13]. They also gave a lower bound for the product dimension of a graph (Theorem 5.3 [13]) which in particular tells that the product dimension of a tree on $n$ vertices with $l$ leaves is at least $\log (n-l+1)$. The authors also suggested that the idea used to encode paths could be extended to study the product dimension of trees. Immediately after this paper, Poljak and Pultr in [16] came up with bounds on product dimension of trees using the encoding for paths employed in [13]. The results in this paper are $\operatorname{pdim}(T) \leqslant 3\lceil\log |T|\rceil$ and $\log |m(T)|-1 \leqslant$ $\operatorname{pdim}(T) \leqslant 3\lceil\log |m(T)|\rceil+1$ where, $T$ is a forest and $m(T)$ is the graph obtained from $T$ by recursively deleting a leaf vertex with one or more siblings. In this paper we improve the above upper bound to $1.441 \log |T|+3$. More recently, in 2010, Ida Kantor in her doctoral thesis [11] determines another upper bound on the product dimension of trees viz. $2+\left\lceil\log \delta_{r}\right\rceil+\sum_{i \in S, 2 \leqslant i<r}\left\lceil\log \delta_{i}\right\rceil+\sum_{i \notin S, 3 \leqslant i<r}\left\lceil\log \left(\delta_{i}-1\right)\right\rceil$, where $r$ is the radius of the tree, $x$ is a central vertex, $\delta_{i}$ is the maximum degree among all vertices which are at a distance $r-i$ from $x$ and $S=\left\{2^{i}: i \in \mathbb{N}\right\}$. The technique used is a generalization of the technique used by Lovász, Nešetřil and Pultr in [13] for paths.

The product dimension of graphs obtained by amalgamation of smaller graphs was studied in [1]. The idea of using orthogonal Latin squares to encode a disjoint union of complete graphs is given by Evans, Isaak and Narayan in [9]. This idea is the motivation for our Amalgamation Lemma for General Graphs (Lemma 7) which is a key ingredient for showing that the product dimension of a graph on $n$ vertices with treewidth at most $t$ is at most $(t+2)(\log n+1)$. Orthogonal Latin squares have been known for a long time. In the 1780s Euler demonstrated methods for constructing orthogonal Latin squares of order $t$ where $t$ is odd or a multiple of 4 and later conjectured that orthogonal Latin squares of order $t \equiv 2 \bmod 4$ do not exist. In 1960, Parker, Bose, and Shrikhande in [3] disproved Euler's conjecture for all $t \geqslant 10$. Thus, orthogonal Latin squares exist for all orders $t \geqslant 3$ except $t=6$. We use this result to prove Lemma 7 . 
A parameter closely related to product dimension of a graph $G$ is the equivalence number of the complement of the graph $G, \bar{G}$. An equivalence is a vertex disjoint union of cliques and the equivalence number of a graph $H$ is the minimum number of equivalences required to cover the edges of $H$. In [2], Alon came up with bounds on the equivalence number of a graph showing $\log n-\log d \leqslant e q(\bar{G}) \leqslant 2 e^{2}(d+1)^{2} \ln n$, where $G$ is a graph on $n$ vertices with maximum degree $d$. It is easy to see that $\operatorname{pdim}(G) \leqslant e q(\bar{G})+1([5])$. Eaton and Rödl in [6] proved that $\operatorname{pdim}(G) \leqslant 32 k \log n$ for a $k$-degenerate graph $G$ on $n$ vertices. Since degeneracy of a graph is at most its maximum degree, this result is a significant improvement over Alon's result. We use a probabilistic method to further improve this upper bound to $\lceil 5.545 k \log n\rceil+1$.

The product dimension of a graph is closely related to the representation number of a graph - a concept introduced by Erdös in [7]. A graph $G$ is representable modulo $r$ if there exists an injection $f: V(G) \rightarrow\{0, \ldots, r-1\}$ such that for all $u, v \in V(G)$, $\operatorname{gcd}(f(u), f(v))=1$ if and only if $\{u, v\} \in E(G)$. The minimum $r$ modulo which $G$ is representable is called the representation number of $G$. The relationship between the two concepts viz. the product dimension of a graph and representation number of a graph is described in [8].

\subsection{Summary of Results}

1. For any forest $T$ on $n$ vertices, $\operatorname{pdim}(T) \leqslant 1.441 \log n+3$ (Theorem 4 ).

This is an improvement over the upper bound for product dimension of trees and forests given by Poljak and Pultr in [16] viz. $3\lceil\log n\rceil$. We use a technique of divide and conquer to prove the theorem. The divide operation corresponds to the operation described in our Splitting Lemma for Forests (Lemma 1) while the conquer operation corresponds to our Amalgamation Lemma for Bipartite Graphs (Lemma 3).

2. For any graph $G$ on $n$ vertices and treewidth $t, \operatorname{pdim}(G) \leqslant(t+2)(\log n+1)$ (Theorem 8).

The techniques used to prove Theorem 4 for trees inspired us to work for graphs with bounded treewidth. Another key ingredient in proving this theorem is the Amalgamation Lemma for General Graphs (Lemma 7) which is based on the existence of orthogonal Latin squares of different orders. Since treewidth $t$ graphs are $t$-degenerate (Section 4.2, [12]), it follows from an upper bound on product dimension based on degeneracy of a graph $[6]$ that $\operatorname{pdim}(G) \leqslant 32 t \log n$. Our result is an improvement over that.

3. For every $k$-degenerate graph $G$ on $n$ vertices, $\operatorname{pdim}(G) \leqslant\lceil 5.545 k \log n\rceil+1$ (Theorem 9).

We derive this result as an improvement over Eaton's and Rödl's upper bound of $32 k \log n$ for product dimension of $k$-degenerate graphs [6]. We use a probabilistic 
argument to prove the theorem and we believe that our proof is shorter.

\subsection{Notations and Definitions}

In this paper we consider only undirected, simple, finite graphs. For any graph $G, V(G)$ denotes its vertex set and $E(G)$ denotes its edge set. The cardinality of a set $S$ is denoted by $|S|$. For a graph $G,|G|$ denotes the cardinality of $V(G) . N_{G}(u)$ denotes the open neighborhood of vertex $u$ in $G$, i.e. all the vertices adjacent to $u$ in $G$. The degree of a vertex $u$, denoted by $d(u)$ is $|N(u)|$.

A subgraph $H$ of $G$ is a graph with vertex set $V(H) \subset V(G)$ and edge set $E(H) \subset$ $E(G) \cap\left\{\left\{v, v^{\prime}\right\}: v, v^{\prime} \in V(H)\right\}$. If $H$ is a subgraph of $G$ then $G$ is called the supergraph of $H$.

For a graph $G$, the graph induced by a set $X \subset V(G)$, denoted by $G[X]$, is the graph with $V(G[X])=X$ and $E(G[X])=E(G) \cap\left\{\left\{v, v^{\prime}\right\}: v, v^{\prime} \in X\right\}$.

If $G_{1}$ and $G_{2}$ are two graphs, then $G_{1} \backslash G_{2}$ is the graph $G_{1}\left[V\left(G_{1}\right) \backslash V\left(G_{2}\right)\right]$. If $G$ is a graph and $S \subset V(G)$, then $G \backslash S$ is the graph $G[V(G) \backslash S]$. The union of two graphs $G_{1}$ and $G_{2}$, denoted by $G_{1} \cup G_{2}$, is the graph with $V\left(G_{1} \cup G_{2}\right)=V\left(G_{1}\right) \cup V\left(G_{2}\right)$ and $E\left(G_{1} \cup G_{2}\right)=E\left(G_{1}\right) \cup E\left(G_{2}\right)$. Moreover, if $V\left(G_{1}\right) \cap V\left(G_{2}\right)=\emptyset$, then we call it a disjoint union and denote it as $G_{1} \uplus G_{2}$. The intersection of two graphs $G_{1}$ and $G_{2}$ is the graph $G_{1} \cap G_{2}$ with $V\left(G_{1} \cap G_{2}\right)=V\left(G_{1}\right) \cap V\left(G_{2}\right)$ and $E\left(G_{1} \cap G_{2}\right)=E\left(G_{1}\right) \cap E\left(G_{2}\right)$.

The graph $G_{1} \times G_{2}$ is the direct product of two graphs $G_{1}$ and $G_{2}$ with $V\left(G_{1} \times G_{2}\right)=$ $V\left(G_{1}\right) \times V\left(G_{2}\right)$ and $E\left(G_{1} \times G_{2}\right)=\left\{\{u, v\}: u, v \in V\left(G_{1}\right) \times V\left(G_{2}\right)\right.$ and if $u=\left(x_{1}, x_{2}\right)$, $v=\left(y_{1}, y_{2}\right)$, then $\left\{x_{1}, y_{1}\right\} \in E\left(G_{1}\right)$ and $\left.\left\{x_{2}, y_{2}\right\} \in E\left(G_{2}\right)\right\}$.

Let $[n]$ denote the set $\{1, \ldots, n\}$. The set of all natural numbers is denoted by $\mathbb{N}$. $\{a\}^{k}$ denotes the $k$-tuple $(a, \ldots, a)$. Throughout the paper, $\log n$ denotes $\log _{2} n$ and $\ln n$ denotes $\log _{e} n$.

\section{Product Dimension of Forests}

Definition 1 (Split vertex). In a forest $F$ on $n$ vertices, a vertex $v$ is called

1. an $(\epsilon, 2)$-split vertex if $F \backslash\{v\}=F_{1} \uplus F_{2}$ such that $\left|F_{1}\right|,\left|F_{2}\right| \leqslant\left(\frac{1}{2}+\epsilon\right) n$, and

2. an $(\epsilon, 3)$-split vertex if $F \backslash\{v\}=F_{1} \uplus F_{2} \uplus F_{3}$ such that $\left|F_{1}\right|,\left|F_{2}\right|,\left|F_{3}\right| \leqslant\left(\frac{1}{2}-\epsilon\right) n$, where $F_{1}, F_{2}$ and $F_{3}$ are subgraphs of $F$.

Lemma 1 (Splitting Lemma for Forests). In every forest $F$, for every $\epsilon \geqslant 0$, there exists either an $(\epsilon, 2)$-split vertex or an $(\epsilon, 3)$-split vertex.

Proof. Let $T$ be a tree such that $V(T)=V(F)$ and $T$ is a supergraph of $F$. It is easy to see that if $v \in V(T)$ is an $(\epsilon, 2)$-split vertex of $T$ with $T \backslash\{v\}=T_{1} \uplus T_{2}$ and $\left|T_{1}\right|,\left|T_{2}\right| \leqslant\left(\frac{1}{2}+\epsilon\right) n$ then $v$ is also an $(\epsilon, 2)$-split vertex of $F$ with $F \backslash\{v\}=F_{1} \uplus F_{2},\left|F_{1}\right|,\left|F_{2}\right| \leqslant\left(\frac{1}{2}+\epsilon\right) n$ where each $F_{i}$ is a subgraph of $T_{i}$. Similarly any $(\epsilon, 3)$-split vertex of $T$ is also an $(\epsilon, 3)$-split 
vertex of $F$. Hence it suffices to show that either an $(\epsilon, 2)$-split vertex or an $(\epsilon, 3)$-split vertex exists in the tree $T$.

Let $n=|T|$. For any $v \in V(T)$, let $C_{1}(v), \ldots, C_{m}(v)$ denote the (connected) components of $T \backslash\{v\}$ such that $\left|C_{1}(v)\right| \geqslant \cdots \geqslant\left|C_{m}(v)\right|$. Choose $v \in V(T)$ such that $\left|C_{1}(v)\right|=\min \left\{\left|C_{1}(u)\right|: u \in V(T)\right\}$. First we claim that $\left|C_{1}(v)\right| \leqslant\left(\frac{1}{2}+\epsilon\right) n$. For the sake of contradiction, let us assume that $\left|C_{1}(v)\right|>\left(\frac{1}{2}+\epsilon\right) n$. Let $w \in C_{1}(v) \cap N_{T}(v)$. If $C_{1}(w) \subset C_{1}(v)$, then $\left|C_{1}(w)\right|<\left|C_{1}(v)\right|$ (because $C_{1}(w) \subset C_{1}(v) \backslash\{v\}$ ) contradicting the choice of $v$. Hence, $C_{1}(w) \subset T \backslash C_{1}(v)$ and $\left|C_{1}(w)\right| \leqslant n-\left|C_{1}(v)\right|<\left(\frac{1}{2}-\epsilon\right) n<\left|C_{1}(v)\right|$. This again contradicts the choice of $v$.

If $\left|C_{1}(v)\right|>\left(\frac{1}{2}-\epsilon\right) n$, then $v$ is an $(\epsilon, 2)$-split vertex and $T_{1}=C_{1}(v), T_{2}=T \backslash\left(T_{1} \cup\{v\}\right)$. Otherwise, let $Q_{1}=C_{1}(v), \ldots, Q_{m}=C_{m}(v)$. Hence, $\left|Q_{i}\right| \leqslant\left(\frac{1}{2}-\epsilon\right) n$ for all $i \in[m]$. If $m \leqslant 3$, then $v$ is either an $(\epsilon, 3)$-split vertex or an $(\epsilon, 2)$-split vertex with $T_{i}=Q_{i}$. If $m \geqslant 4$, consider a partition $I_{1} \uplus \ldots \uplus I_{k}=[m]$ with minimum possible $k$ such that $\left|\cup_{j \in I_{l}} Q_{j}\right| \leqslant\left(\frac{1}{2}-\epsilon\right) n$ for all $l \in[k]$. For $k \leqslant 3, v$ is either an $(\epsilon, 2)$-split vertex or an $(\epsilon, 3)$-split vertex with $T_{l}=\cup_{j \in I_{l}} Q_{j}$. Suppose $k \geqslant 4$, define $Q_{l}^{\prime}=\cup_{j \in I_{l}} Q_{j}, l \in[k]$ and let $Q^{\prime}$ be the union of smallest two among $\left\{Q_{1}^{\prime}, \ldots, Q_{k}^{\prime}\right\}$. Hence, $\left|Q^{\prime}\right| \leqslant \frac{n}{2} \leqslant\left(\frac{1}{2}+\epsilon\right) n$ by the pigeonhole principle. By the minimality in the choice of the partition $I_{1} \uplus \ldots \uplus I_{k}$, $\left|Q^{\prime}\right|>\left(\frac{1}{2}-\epsilon\right) n$. Thus, $v$ is an $(\epsilon, 2)$-split vertex with $T_{1}=Q^{\prime}$ and $T_{2}=T \backslash\left(Q^{\prime} \cup\{v\}\right)$.

Definition 2. We call an l-encoding $\phi_{G}$ of a graph $G$, a well-begun l-encoding if the first coordinate of $\phi_{G}$ is from $\{0, \ldots, \chi(G)-1\}$.

Observation 2. For any $q>p$, if $\phi_{G}$ is a p-encoding of $G$, then $\psi_{G}$, obtained from $\phi_{G}$ by adding $q-p$ coordinates to $\phi_{G}$ such that for all $p<i \leqslant q$, the $i$-th coordinate of $\psi_{G}(x)$ is the $p$-th coordinate of $\phi_{G}$, is a q-encoding of $G$.

Lemma 3 (Amalgamation Lemma for Bipartite Graphs). Let $G_{0}, \ldots, G_{k-1}$ be bipartite graphs such that $G_{i} \cap G_{j}=\{g\}$ for all $i, j \in\{0, \ldots, k-1\}, i \neq j$. Let $G=\cup_{i=0}^{k-1} G_{i}$. For every $i \in\{0, \ldots, k-1\}$, let $\phi_{G_{i}}$ be a well-begun $l_{i}$-encoding of $G_{i}$. Then we can construct a well-begun $l$-encoding $\phi_{G}$ of $G$, where $l=\max _{0 \leqslant i \leqslant k-1}\left\{l_{i}\right\}+\lceil\log k\rceil$.

Proof. From Observation 2, without loss of generality we can assume that $l_{0}=\cdots=$ $l_{k-1}=\max _{i}\left\{l_{i}\right\}$. Since we can rename the alphabets used in each coordinate of an encoding independently of the other coordinates, it is safe to assume that the vertex $g$ gets the encoding $\{0\}^{l_{0}}$ in every $\phi_{G_{i}}$. For all $0 \leqslant i \leqslant k-1$, let $b_{0}(i)$ denote the binary representation of $i$ using exactly $\lceil\log k\rceil$ bits and $b_{1}(i)$ denote the bitwise complement of $b_{0}(i)$. The $l$-encoding $\phi_{G}$ of $G$ is as follows.

For all $i, 0 \leqslant i \leqslant k-1$, for every $x \in V\left(G_{i} \backslash\{g\}\right)$

$$
\begin{aligned}
& \phi_{G}(x)= \begin{cases}\phi_{G_{i}}(x) b_{0}(i) & \text { if } \phi_{G_{i}}(x) \text { begins with } 0 \\
\phi_{G_{i}}(x) b_{1}(i) & \text { if } \phi_{G_{i}}(x) \text { begins with } 1\end{cases} \\
& \phi_{G}(g)=\{0\}^{l_{0}}\{2\}^{\lceil\log k\rceil}
\end{aligned}
$$

We can verify that $\phi_{G}$ is a valid $l$-encoding of $G$ from the following argument. 


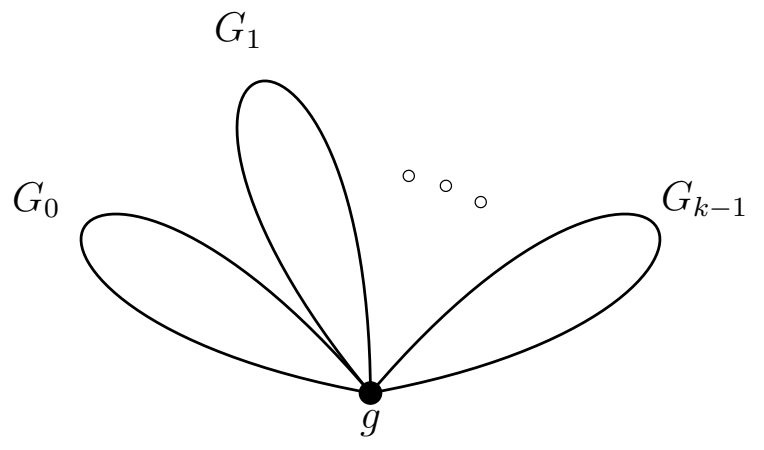

Figure 1: A graph $G=\cup_{i=0}^{k-1} G_{i}$ where $G_{i} \cap G_{j}=\{g\}$ for all $i, j \in\{0, \ldots, k-1\}, i \neq j$.

Let $x, y \in V\left(G_{i} \backslash\{g\}\right)$. If $\{x, y\} \in E\left(G_{i}\right)$ then the first coordinates of $\phi_{G_{i}}(x)$ and $\phi_{G_{i}}(y)$ are different. Thus, the extra coordinates added to $\phi_{G_{i}}(x)$ and $\phi_{G_{i}}(y)$ to get $\phi_{G}(x)$ and $\phi_{G}(y)$ are complements of each other (by Equation (1)). If $\{x, y\} \notin E\left(G_{i}\right)$, then $\phi_{G_{i}}(x)$ and $\phi_{G_{i}}(y)$ agreed in some coordinate, say $t$. Hence, $\phi_{G}(x)$ and $\phi_{G}(y)$ also agree in the $t$-th coordinate.

Let $x \in V\left(G_{i} \backslash\{g\}\right)$ and $y \in V\left(G_{j} \backslash\{g\}\right)$ for some $i, j \in\{0, \ldots, k-1\}, i \neq j$. Note that, since $G_{i} \cap G_{j}=\{g\},\{x, y\} \notin E(G)$. If $\phi_{G_{i}}(x)$ and $\phi_{G_{i}}(y)$ agree in the first coordinate then $\phi_{G}(x)$ and $\phi_{G}(y)$ also agree in the first coordinate. If $\phi_{G_{i}}(x)$ begins with 0 and $\phi_{G_{i}}(y)$ begins with 1 , then $\phi_{G}(x)=\phi_{G_{i}}(x) b_{0}(i)$ and $\phi_{G}(y)=\phi_{G_{j}}(y) b_{1}(j)$. Since $i \neq j, b_{0}(i)$ and $b_{1}(j)$ agree in some coordinate.

For any $i$, let $x \in V\left(G_{i} \backslash\{g\}\right)$. If $\{g, x\} \notin E\left(G_{i}\right)$, then $\phi_{G_{i}}(g)$ and $\phi_{G_{i}}(x)$ agreed in some coordinate, say $t$. Hence, $\phi_{G}(g)$ and $\phi_{G}(x)$ also agree in the $t$-th coordinate. In the other case, that is, when $\{g, x\} \in E\left(G_{i}\right)$, since $\phi_{G_{0}}(g)$ begins with $0, \phi_{G_{i}}(x)$ must begin with 1 . Thus, the extra coordinates added to $\phi_{G_{i}(x)}$ to get $\phi_{G}(x)$ are $b_{1}(i)$ while the extra coordinates added to $\phi_{G_{0}}(g)$ to get $\phi_{G}(g)$ are $\{2\}^{\lceil\log k\rceil}$. Therefore, $\phi_{G}(x)$ and $\phi_{G}(g)$ disagree in all coordinates.

It is easy to see from Equation 1 that $\phi(G)$ is well-begun.

Theorem 4. For any forest $T$ on $n$ vertices, $\operatorname{pdim}(T) \leqslant 1.441 \log n+3$.

Proof. Let $V(T)=\left\{v_{0}, \ldots, v_{n-1}\right\}, f: V(T) \longrightarrow\{0,1, \ldots, n-1\}$ be a bijection, and $f_{i}=$ $f\left(v_{i}\right)$. We use a divide and conquer strategy to prove the theorem. Let $C(T)$ denote the minimum $l$ such that there exists a well-begun $l$-encoding of $T$. Let $C(n)=\max \{C(T): T$ is a forest on at most $n$ vertices $\}$.

Base Case. All possible forests with $|V(T)| \leqslant 3$ with their well-begun 3-encodings are shown in Figure 2. Thus, $C(3) \leqslant 3$.

Note that the third coordinate of each of the encodings is always a unique number associated with the vertex. This ensures injectivity of all the encodings that we get during the conquer steps. 


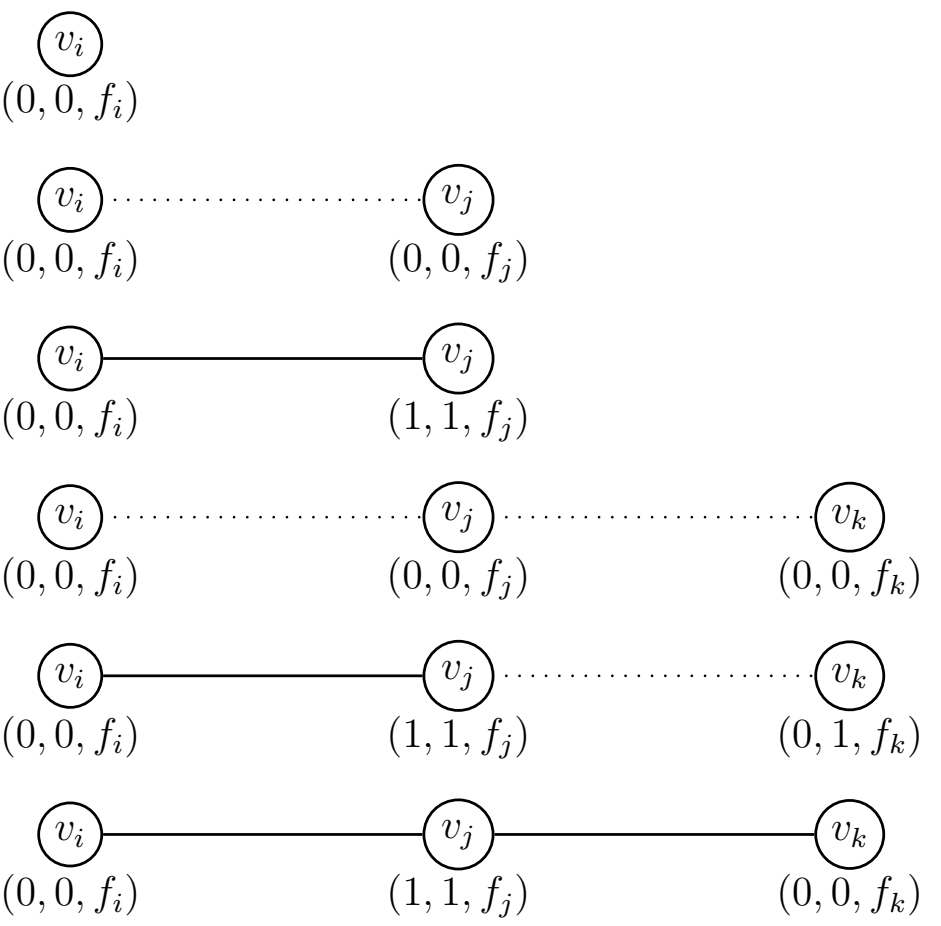

Figure 2: Well-begun 3-encodings of the six forests with at most 3 vertices. Each row depicts a single forest and dotted lines are non-edges.

Divide and Conquer. In our divide and conquer strategy, the divide operation corresponds to the two splitting operations of Lemma 1 viz. $(\epsilon, 2)$-splitting and $(\epsilon, 3)$-splitting and the conquer operation corresponds to the amalgamation operation of Lemma 3.

Choose $\epsilon=\frac{\sqrt{5}}{2}-1$. Let $\alpha=\frac{1}{2}+\epsilon$ and $\beta=\frac{1}{2}-\epsilon$. Note that $\alpha^{2}=\beta$. By Lemma 1 , there exists either an $(\epsilon, 2)$-split vertex or an $(\epsilon, 3)$-split vertex, say $v \in V(T)$. If $v$ is an $(\epsilon, 2)$-split vertex, then from Definition $1, T \backslash\{v\}=T_{1} \uplus T_{2}$ such that $\left|T_{1}\right|,\left|T_{2}\right| \leqslant \alpha n$. Let $T_{i}^{\prime}=T_{i} \cup\{v\}, i \in[2]$. Let $\phi_{T_{i}^{\prime}}$ be a well-begun $l_{i}$-encoding of $T_{i}^{\prime}, i \in[2]$. Then by Lemma 3 , there exists a well-begun $l$-encoding $\phi_{T}$ of $T$ with $l=\max \left\{l_{1}, l_{2}\right\}+1$. Similarly, if $v$ is an $(\epsilon, 3)$-split vertex, then from Definition $1, T \backslash\{v\}=T_{1} \uplus T_{2} \uplus T_{3}$ such that $\left|T_{1}\right|,\left|T_{2}\right|,\left|T_{3}\right| \leqslant \beta n$. Let $T_{i}^{\prime}=T_{i} \cup\{v\}, i \in[3]$. Let $\phi_{T_{i}^{\prime}}$ be a well-begun $l_{i^{-} \text {-encoding }}$ of $T_{i}^{\prime}, i \in[3]$. Then by Lemma 3 , there exists a well-begun l-encoding $\phi_{T}$ of $T$ with $l=\max \left\{l_{1}, l_{2}, l_{3}\right\}+2$.

Therefore, the following recurrence relation holds.

$$
\begin{aligned}
& C(n) \leqslant \max \{C(\alpha n+1)+1, C(\beta n+1)+2\} \\
& C(3) \leqslant 3
\end{aligned}
$$

Solving the recurrence. Let $X$ be an arbitrary leaf in the recurrence tree and let $P$ denote the path from the root to $X$. Let the number of $(\epsilon, 2)$-split operations and $(\epsilon, 3)$ - 
split operations along $P$ be $k_{2}$ and $k_{3}$ respectively. Let $s_{i}$ be the size of the subgraph of $T$ to be conquered along $P$ after $i$ steps. Let $\gamma_{1}, \ldots, \gamma_{k}, k=k_{2}+k_{3}$, be such that

$$
\gamma_{i}= \begin{cases}\alpha & \text { if the } i \text {-th divide operation along } P \text { is an }(\epsilon, 2) \text {-split operation } \\ \beta & \text { if the } i \text {-th divide operation along } P \text { is an }(\epsilon, 3) \text {-split operation }\end{cases}
$$

Therefore, $s_{k} \leqslant\left(\prod_{j=1}^{k} \gamma_{j}\right) n+\prod_{j=2}^{k} \gamma_{j}+\prod_{j=3}^{k} \gamma_{j}+\ldots+\prod_{j=k}^{k} \gamma_{j}+1$. Since $\gamma_{i} \leqslant \alpha$ for all $i, 1 \leqslant i \leqslant k, s_{k} \leqslant\left(\prod_{j=1}^{k} \gamma_{j}\right) n+\alpha^{k-1}+\alpha^{k-2}+\ldots+\alpha+1 \leqslant \alpha^{k_{2}} \beta^{k_{3}} n+\frac{1}{1-\alpha} \leqslant \alpha^{k_{2}} \beta^{k_{3}} n+2.62$. Hence, $s_{k} \leqslant\left\lfloor\alpha^{k_{2}+2 k_{3}} n+2.62\right\rfloor$. Note that $k_{2}+2 k_{3}$ is the total cost of conquering (number of coordinates introduced by the amalgamation operation) incurred along $P$. Since $X$ is arbitrary, $C(n) \leqslant k_{2}+2 k_{3}+C\left(s_{k}\right)$.

Let $k_{2}+2 k_{3} \geqslant 1.441 \log n$. Then $s_{k} \leqslant 3$. Hence, $C(n) \leqslant 1.441 \log n+C(3) \leqslant$ $1.441 \log n+3$. Therefore, $\operatorname{pdim}(T) \leqslant 1.441 \log n+3$.

\section{Product Dimension of Bounded Treewidth Graphs}

Definition 3 (Definition 1, [4]). A tree decomposition of $G$ is a pair $\left(\left\{X_{i}: i \in I\right\}, T\right)$, where $I$ is an index set, $\left\{X_{i}: i \in I\right\}$ is a collection of subsets of $V(G)$ and $T$ is a tree whose node set is $I$, such that the following conditions are satisfied:

1. $\cup_{i \in I} X_{i}=V(G)$.

2. $\forall\{u, v\} \in E(G), \exists i \in I$ such that $u, v \in X_{i}$.

3. $\forall i, j, k \in I$ : if $j$ is on a path in $T$ from $i$ to $k$, then $X_{i} \cap X_{k} \subset X_{j}$.

The width of a tree decomposition $\left(\left\{X_{i}: i \in I\right\}, T\right)$ is $\max \left\{\left|X_{i}\right|: i \in I\right\}-1$. The treewidth of $G, t w(G)$, is the minimum width over all tree decompositions of $G$.

Note that by a rooted tree we mean a tree with a vertex designated as the root vertex.

Definition 4 (Definition 2, [4]). A normalized tree decomposition of a graph $G$ is a triple $\left(\left\{X_{i}: i \in I\right\}, r \in I, T\right)$ where $\left(\left\{X_{i}: i \in I\right\}, T\right)$ is a tree decomposition of $G$ that additionally satisfies the following two properties:

4. It is a rooted tree where the subset $X_{r}$ that corresponds to the root node $r$ contains exactly one vertex.

5. For any node $i$, if $i^{\prime}$ is the child of $i$, then $\left|X_{i^{\prime}} \triangle X_{i}\right|=1$ where, $X_{i^{\prime}} \triangle X_{i}$ denoted the symmetric difference of $X_{i^{\prime}}$ and $X_{i}$.

Lemma 5 (Lemma 3, [4]). For any graph $G$ there is a normalized tree decomposition with width equal to tw $(G)$. 
Lemma 6 (Splitting Lemma for Bounded Treewidth Graphs). Let $G$ be a graph on $n$ vertices with $t w(G)=t$ and a normalized tree decomposition $\left(\left\{X_{i}: i \in I\right\}, r \in I, T\right)$ of width $t$. Then there exists $l \in I$ such that $G \backslash X_{l}=G_{1} \uplus G_{2} \uplus G_{3}$ and $\left|G_{i}\right| \leqslant \frac{1}{2}\left(n-\left|X_{l}\right|+1\right)$, $i \in[3]$, where $G_{1}, G_{2}$ and $G_{3}$ are subgraphs of $G$.

Proof. For every $i$, let $D_{1}(i), \ldots, D_{t}(i)$ be the components of $T \backslash\{i\}$ and let $C_{l}(i), j \in[t]$, be the graphs induced by $\left(\cup_{j \in V\left(D_{l}(i)\right)} X_{j}\right) \backslash X_{i}$. Without loss of generality assume that $\left|C_{1}(i)\right| \geqslant \cdots \geqslant\left|C_{t}(i)\right|$.

Let $c=\min \left\{\left|C_{1}(j)\right|: j \in I\right\}$ and $I^{\prime}=\left\{j \in I:\left|C_{1}(j)\right|=c\right\}$. Then choose $l \in I^{\prime}$ such that $\left|X_{l}\right|=\min \left\{\left|X_{j}\right|: j \in I^{\prime}\right\}$. We claim that, $\left|C_{1}(l)\right| \leqslant \frac{1}{2}\left(n-\left|X_{l}\right|+1\right)$. For the sake of contradiction, assume that $\left|C_{1}(l)\right|>\frac{1}{2}\left(n-\left|X_{l}\right|+1\right)$. Let $m \in N_{T}(l) \cap D_{1}(l)$. Then, since $T$ is a normalized tree decomposition $\left|X_{m} \triangle X_{l}\right|=1$, therefore, the following two cases arise.

Case $1\left(X_{m}=X_{l} \cup\{v\}\right.$ where $\left.v \in V(G)\right)$.

If $D_{1}(m) \subset D_{1}(l)$, then $\left|C_{1}(m)\right|<\left|C_{1}(l)\right|$ because $C_{1}(m)=C_{1}(l) \backslash\{v\}$. Otherwise, $D_{1}(m)=T \backslash D_{1}(l)$ in which case $\left|C_{1}(m)\right|=\left|G \backslash\left(C_{1}(l) \cup X_{l}\right)\right|=n-\left|C_{1}(l)\right|-\left|X_{l}\right|<$ $n-\frac{1}{2}\left(n-\left|X_{l}\right|+1\right)-\left|X_{l}\right|=\frac{1}{2}\left(n-\left|X_{l}\right|-1\right)<\left|C_{1}(l)\right|$. In either case, $\left|C_{1}(m)\right|<\left|C_{1}(l)\right|$, contradicting the choice of $l$.

Case $2\left(X_{m}=X_{l} \backslash\{v\}\right.$ where $\left.v \in V(G)\right)$.

If $D_{1}(m) \subset D_{1}(l)$, then $\left|C_{1}(m)\right| \leqslant\left|C_{1}(l)\right|$. If $\left|C_{1}(m)\right|<\left|C_{1}(l)\right|$, then $\left|C_{1}(m)\right|$ is not the minimum amongst all $\left|C_{1}(j)\right|, j \in I$ and if $\left|C_{1}(m)\right|=\left|C_{1}(l)\right|$ then, since $\left|X_{m}\right|<\left|X_{l}\right|$, the choice of $l$ is contradicted. On the other hand, if $D_{1}(m)=T \backslash D_{1}(l)$, then $\left|C_{1}(m)\right|=$ $\left|G \backslash\left(C_{1}(l) \cup X_{m}\right)\right|=n-\left|C_{1}(l)\right|-\left|X_{m}\right|<n-\frac{1}{2}\left(n-\left|X_{l}\right|+1\right)-\left|X_{l}\right|+1=\frac{1}{2}\left(n-\left|X_{l}\right|+1\right)$ again contradicting the choice of $l$.

Hence $C_{1}(l) \leqslant \frac{1}{2}\left(n-\left|X_{l}\right|+1\right)$ i.e., $G \backslash X_{l}=C_{1}(l) \uplus \cdots \uplus C_{t}(l)$ such that $\left|C_{j}(l)\right| \leqslant$ $\frac{1}{2}\left(n-\left|X_{l}\right|+1\right)$ for all $j \in[t]$.

Consider a partition $I_{1} \uplus \cdots \uplus I_{r}=[t]$ with minimum possible $r$ such that $\left|\cup_{j \in I_{i}} C_{j}(l)\right| \leqslant$ $\frac{1}{2}\left(n-\left|X_{l}\right|+1\right)$ for all $i \in[r]$. Let $\cup_{j \in I_{i}} C_{j}(l)=H_{i}$ for all $i \in[r]$. Rename all $H_{i}$ 's such that $\left|H_{1}\right| \geqslant \cdots \geqslant\left|H_{r}\right|$. We claim that for such a partition $r \leqslant 3$ because if $r \geqslant 4$ then $\left|\cup_{j=\left\lceil\frac{r}{2}\right\rceil+1}^{r} H_{j}\right| \leqslant \frac{1}{2}\left(n-\left|X_{l}\right|\right)$ by the pigeonhole principle contradicting the choice of the partition $I_{1} \uplus \cdots \uplus I_{r}$. Set $G_{i}=H_{i}$ for $i \in[3]$ and we are done.

Lemma 7 (Amalgamation Lemma for General Graphs). Let $G=G_{1} \cup G_{2} \cup G_{3}$ where $G_{1}, G_{2}$ and $G_{3}$ are graphs such that $G_{i} \cap G_{j}=S$ for all $i, j \in[3]$ and $i \neq j$. Let $G_{1}^{\prime}, G_{2}^{\prime}$ and $G_{3}^{\prime}$ be graphs such that $V\left(G_{i}^{\prime}\right)=V\left(G_{i}\right)$ and $E\left(G_{i}^{\prime}\right)=E\left(G_{i}\right) \cup\left\{\left\{v, v^{\prime}\right\}: v, v^{\prime} \in V(S)\right\}$ for all $i \in[3]$. Let $\phi_{G_{i}^{\prime}}$ be an $l_{i}$-encoding of $G_{i}^{\prime}$ for all $i \in[3]$ and $\phi_{S}$ be an $l_{s}$-encoding of $S$. Then we can construct an l-encoding of $G$, where

$$
l= \begin{cases}\max \left\{l_{1}, l_{2}, l_{3}\right\}+\max \left\{\chi(G \backslash S)+1, l_{s}\right\} & \text { if } \chi(G \backslash S)=2 \text { or } 6 \\ \max \left\{l_{1}, l_{2}, l_{3}\right\}+\max \left\{\chi(G \backslash S), l_{s}\right\} & \text { otherwise }\end{cases}
$$




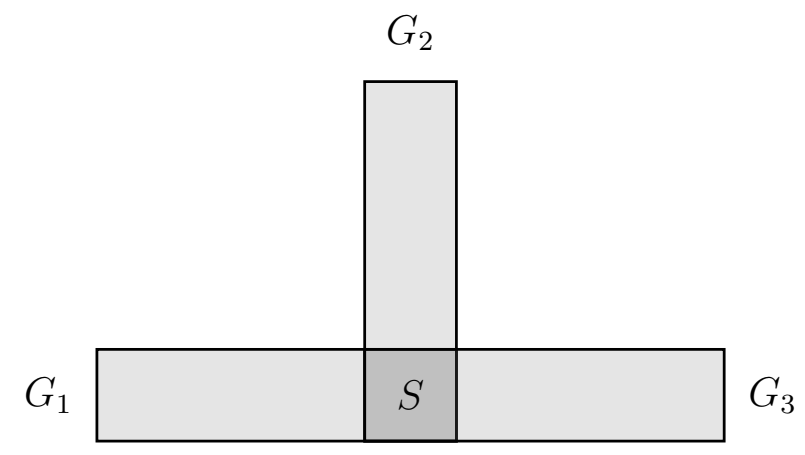

Figure 3: A graph $G=\cup_{i=1}^{3} G_{i}$ where $G_{i} \cap G_{j}=S$ for all $i, j \in[3]$ and $i \neq j$

Proof. Without loss of generality we can assume that the alphabets used in $\phi_{S}$ are disjoint from the alphabets used in $\phi_{G_{i}^{\prime}}$ for all $i \in[3]$ and greater than $\chi(G)$, and also from Observation 2 , let $l_{1}=l_{2}=l_{3}=\max \left\{l_{1}, l_{2}, l_{3}\right\}$. Let $V(G)=\left\{v_{0}, \ldots, v_{n-1}\right\}, f: V(G) \rightarrow$ $\{0, \ldots, n-1\}$ be a bijection, and $f_{i}=f\left(v_{i}\right)$. Let us rename the alphabets in each coordinate of $\phi_{G_{i}^{\prime}}$ such that all $v_{j} \in V(S)$ get the encoding as $\left(f_{j}, \ldots, f_{j}\right)$ for all $i \in[3]$.

Let $c: V(G \backslash S) \rightarrow\{0, \ldots, \chi(G \backslash S)-1\}$ be an optimal proper coloring of the vertices $V(G \backslash S)$.

Let

$$
t= \begin{cases}\chi(G \backslash S)+1 & \text { if } \chi(G \backslash S)=2 \text { or } 6 \\ \chi(G \backslash S) & \text { otherwise }\end{cases}
$$

By Theorem 4.3 in [9], if we have two orthogonal Latin squares of order $t$, we can have a $t$-encoding for $3 K_{t}$ and hence, for $3 K_{\chi(G \backslash S)}$ as well. Let the $j$-th vertex in the $i$-th copy of $3 K_{\chi(G \backslash S)}$ get the encoding $\phi_{K}(i, j)$ for all $i \in[3]$ and $j \in[\chi(G \backslash S)]$. Note that $\phi_{K}(i, j)$ and $\phi_{K}\left(i, j^{\prime}\right), j \neq j^{\prime}$ disagree at all coordinates and $\phi_{K}(i, j)$ and $\phi_{K}\left(i^{\prime}, j^{\prime}\right), i \neq i^{\prime}$, agree in at least one coordinate, for all $i, i^{\prime} \in[3]$ and $j, j^{\prime} \in[\chi(G \backslash S)]$. Let $m=\max \left\{t, l_{s}\right\}$. From Observation 2, let $\phi_{S}$ and $\phi_{K}(i, j)$ be $m$-encodings of $S$ and $3 K_{\chi(G \backslash S)}$ respectively. We construct an $l$-encoding of $G, \phi_{G}$, is as follows.

$$
\phi_{G}(x)= \begin{cases}\phi_{G_{i}^{\prime}}(x) \phi_{K}(i, c(x)) & \text { if } x \in G_{i} \backslash S \\ \phi_{G_{1}^{\prime}}(x) \phi_{S}(x) & x \in S\end{cases}
$$

We can verify that $\phi_{G}$ is a valid encoding of $G$ from the following argument. Let $x, y \in$ $V\left(G_{i} \backslash S\right)$. If $\{x, y\} \notin E(G)$, then $\{x, y\} \notin E\left(G_{i}^{\prime}\right)$. Therefore, $\phi_{G_{i}^{\prime}}(x)$ and $\phi_{G_{i}^{\prime}}(y)$ agree in some coordinate, say $g$ and thus, $\phi_{G}(x)$ and $\phi_{G}(y)$ also agree in the $g$-th coordinate. If $\{x, y\} \in E(G)$, then $\{x, y\} \in E\left(G_{i}^{\prime}\right)$. Hence, $\phi_{G_{i}^{\prime}}(x)$ and $\phi_{G_{i}^{\prime}}(y)$ do not agree in any coordinate and since, $c$ is a proper coloring of $G \backslash S, c(x) \neq c(y)$. Thus, $\phi_{K}(i, c(x))$ and $\phi_{K}(i, c(y))$ do not agree in any coordinate. Therefore, $\phi_{G}(x)$ and $\phi_{G}(y)$ do not agree in any coordinate. 
Let $x \in V\left(G_{i} \backslash S\right)$ and $y \in V\left(G_{i^{\prime}} \backslash S\right), i \neq i^{\prime}$. Note that $\{x, y\} \notin E(G)$. Since $\phi_{K}(i, c(x))$ and $\phi_{K}\left(i^{\prime}, c(y)\right), i \neq i^{\prime}$ agree in some coordinate, say $g, \phi_{G}(x)$ and $\phi_{G}(y)$ will agree in the $\left(l_{1}+g\right)$-th coordinate.

For any $i$, let $x \in V\left(G_{i} \backslash S\right)$ and $y \in V(S)$. Since $\phi_{S}(y)$ uses new alphabets greater than $\chi_{G}, \phi_{G}(x)$ and $\phi_{G}(y)$ agree in some coordinate if and only if $\phi_{G_{i}^{\prime}}(x)$ and $\phi_{G_{1}^{\prime}}(y)$ $\left(=\phi_{G_{i}^{\prime}}(y)\right)$ agree in some coordinate.

For $x, y \in V(S)$, if $\{x, y\} \in E(G)$, then since $\phi_{G_{1}^{\prime}}(x)=(f(x), \ldots, f(x)), \phi_{G_{1}^{\prime}}(y)=$ $(f(y), \ldots, f(y))$ where $f$ is a bijection and $\phi_{S}(x)$ and $\phi_{S}(y)$ disagree in all coordinates, $\phi_{G}(x)$ and $\phi_{G}(y)$ disagree in all coordinates. If $\{x, y\} \notin E(G)$, then $\{x, y\} \notin E(S)$, Thus, $\phi_{S}(x)$ and $\phi_{S}(y)$ agree in some coordinate, say $g$ and therefore, $\phi_{G}(x)$ and $\phi_{G}(y)$ agree in the $\left(l_{1}+g\right)$-th coordinate.

Theorem 8. For any graph $G$ on $n$ vertices and $t w(G)=t, \operatorname{pdim}(G) \leqslant(t+2)(\log n+1)$.

Proof. We use a divide and conquer strategy to prove the theorem. Let $\mathcal{G}_{t}(n)$ denote the set of all $n$-vertex graphs with treewidth at most $t$ and let $C_{t}(n)=\max \{\operatorname{pdim}(G): G \in$ $\left.\mathcal{G}_{t}(n)\right\}$.

Base case. By Theorem 4.3 in [13], $C_{t}(t+3)=t+2$.

Divide and conquer. In our divide and conquer strategy, the divide operation corresponds to the splitting operation of Lemma 6 and the conquer operation corresponds to the amalgamation operation of Lemma 7.

By Lemma 6, for a graph $G$ on $n$ vertices with $t w(G)=t$ and a normalized tree decomposition $\left(\left\{X_{i}: i \in I\right\}, r \in I, T\right)$ of width $t$, there exists $l \in I$ such that $G \backslash X_{l}=$ $G_{1} \uplus G_{2} \uplus G_{3},\left|G_{i}\right| \leqslant \frac{1}{2}\left(n-\left|X_{l}\right|+1\right), i \in[3]$. Let $G_{i}^{\prime}=G_{i} \cup G\left[X_{l}\right]$ for all $i \in[3]$. Therefore, $\left|G_{i}^{\prime}\right| \leqslant \frac{1}{2}\left(n-\left|X_{l}\right|+1\right)+\left|X_{l}\right|=\frac{1}{2}\left(n+\left|X_{l}\right|+1\right)$ for all $i \in[3]$. Let $\alpha=\frac{1}{2}$ and $\beta=\frac{\left|X_{l}\right|+1}{2}$. Hence, $\left|G_{i}^{\prime}\right| \leqslant \alpha n+\beta$ for all $i \in[3]$.

Let $S=G\left[X_{l}\right]$. Note that $G_{i}^{\prime} \cap G_{j}^{\prime}=S$ for all $i, j \in[3]$ and $i \neq j$, and $G=G_{1}^{\prime} \cup G_{2}^{\prime} \cup G_{3}^{\prime}$. Let $G_{1}^{\prime \prime}, G_{2}^{\prime \prime}, G_{3}^{\prime \prime}$ be graphs such that $V\left(G_{i}^{\prime \prime}\right)=V\left(G_{i}^{\prime}\right)$ and $E\left(G_{i}^{\prime \prime}\right)=E\left(G_{i}^{\prime}\right) \cup\left\{\left\{v, v^{\prime}\right\}: v, v^{\prime} \in\right.$ $V(S)\}$ for all $i \in[3]$ (note that $\left|G_{i}^{\prime \prime}\right| \leqslant \alpha n+\beta, i \in[3]$ ). Let $\phi_{G_{i}^{\prime \prime}}$ is an $l_{i}$-encoding of $G_{i}^{\prime \prime}$ for all $i \in[3]$ and $\phi_{S}$ be an $l_{s}$-encoding of $S$. Then, by Lemma 7 , we can construct an l-encoding of $G$ where

$$
l= \begin{cases}\max \left\{l_{1}, l_{2}, l_{3}\right\}+\max \left\{\chi(G \backslash S)+1, l_{s}\right\} & \text { if } \chi(G \backslash S)=2 \text { or } 6 \\ \max \left\{l_{1}, l_{2}, l_{3}\right\}+\max \left\{\chi(G \backslash S), l_{s}\right\} & \text { otherwise. }\end{cases}
$$

Since $G$ is a graph with $t w(G)=t, \chi(G) \leqslant t+1$ (Theorem 6, [14]), and hence $\chi(G \backslash S) \leqslant$ $t+1$. Also, since $|V(S)| \leqslant t+1$, by Theorem $4.3[13], l_{s} \leqslant t+1$. Therefore,

$$
l \leqslant \begin{cases}\max \left\{l_{1}, l_{2}, l_{3}\right\}+\max \{t+2, t+1\} & \text { if } \chi(G \backslash S)=2 \text { or } 6 \\ \max \left\{l_{1}, l_{2}, l_{3}\right\}+\max \{t+1, t+1\} & \text { otherwise }\end{cases}
$$

Hence,

$$
l \leqslant \max \left\{l_{1}, l_{2}, l_{3}\right\}+t+2
$$


Let $G^{\prime}$ be the graph such that $V\left(G^{\prime}\right)=V(G)$ and $E\left(G^{\prime}\right)=E(G) \cup\left\{\left\{v, v^{\prime}\right\}: v, v^{\prime} \in\right.$ $V(S)\}$. Note that $\left(\left\{X_{i}: i \in I\right\}, r \in I, T\right)$ is a tree decomposition for $G^{\prime}$ too since all the new edges added are between the vertices of the same node. Also since $G \subset G^{\prime}$, $t w(G) \leqslant t w\left(G^{\prime}\right)$. Hence, $t w\left(G^{\prime}\right)=t$ and thus, $t w\left(G_{i}^{\prime \prime}\right) \leqslant t$ (since $G_{i}^{\prime \prime} \subset G^{\prime}$ ) for all $i \in[3]$.

Therefore, the following recurrence relation holds.

$$
\begin{aligned}
C_{t}(n) & \leqslant C_{t}(\alpha n+\beta)+t+2 \\
C_{t}(t+3) & \leqslant t+2
\end{aligned}
$$

Solving the recurrence. Let $X$ be an arbitrary leaf in the recurrence tree and let $P$ denote the path from root to $X$. Let the number of divide operations along $P$ be $d$. Let $s_{j}$ be the size of the subgraph of $G$ to be conquered along $P$ after $j$ steps.

Therefore, $s_{d} \leqslant \alpha^{d} n+\alpha^{d-1} \beta+\alpha^{d-2} \beta+\cdots+\alpha \beta+\beta \leqslant \alpha^{d} n+\frac{\beta}{1-\alpha}=\alpha^{d} n+\left|X_{l}\right|+1 \leqslant$ $\alpha^{d} n+t+2$ (since $\left.\left|X_{l}\right| \leqslant t+1\right)$. Hence, $s_{d} \leqslant\left\lfloor\alpha^{d} n+t+2\right\rfloor$. Note that the total cost of conquering incurred along $P$ is $(t+2) d$.

Let $d \geqslant \log n$. Then $s_{d} \leqslant t+3$. Hence, $C_{t}(n) \leqslant(t+2) \log n+C_{t}(t+3) \leqslant(t+2) \log n+$ $t+2=(t+2)(\log n+1)$. Therefore, $\operatorname{pdim}(G) \leqslant(t+2)(\log n+1)$.

\section{Product Dimension of $k$-degenerate Graphs}

A graph $G$ is called $k$-degenerate if there exists an ordering $v_{1}, \ldots, v_{n}$ of $V(G)$ such that $\left|N\left(v_{i}\right) \cap\left\{v_{j}: j<i\right\}\right| \leqslant k, \forall i \in[n]$. Under an ordering $v_{1}, \ldots, v_{n}$ of $V(G)$, the set $N_{G}\left(v_{i}\right) \cap\left\{v_{j}: j<i\right\}$ is called the set of backward neighbors of $v_{i}$.

Theorem 9. For every $k$-degenerate $\operatorname{graph} G, \operatorname{pdim}(G) \leqslant\lceil 5.545 k \log n\rceil+1$.

Proof. Recall that the product dimension of a graph $G$ is the minimum number of proper colorings of $G$ such that any pair of non-adjacent vertices get the same color in at least one of the colorings and not in all of them.

We use probabilistic arguments to prove the theorem. Let us describe a random coloring procedure using $3 k$ colors for the vertices of $G$. Let $C=[3 k]$ be the set of colors. Let $v_{1}, \ldots, v_{n}$ be an ordering of the vertex set of $G$ such that $\left|N\left(v_{i}\right) \cap\left\{v_{j}: j<i\right\}\right| \leqslant k$. We color the vertices starting from $v_{1}$ such that any vertex $v_{i}$ is assigned a color independently and uniformly at random from the set $C \backslash C_{i}$, where $C_{i}$ is the set of colors used by the backward neighbors of $v_{i}$. Note that $0 \leqslant\left|C_{i}\right| \leqslant k$. It is clear that the coloring thus obtained is proper. Repeat this procedure independently $r$ times to get $r$ random proper colorings of $G$.

For $\left\{v_{i}, v_{j}\right\} \notin E(G)$, let us calculate the probability that both $v_{i}$ and $v_{j}$ get the same color in a particular coloring. Let $C^{\prime}=C \backslash\left(C_{i} \cup C_{j}\right)$. Then the probability $p$ that both $v_{i}$ and $v_{j}$ get the same color in a particular coloring is equal to the probability that $v_{i}$ chooses a color from the set $C^{\prime}$ and $v_{j}$ chooses the same color as chosen by $v_{i}$. Hence $p=\frac{\left|C^{\prime}\right|}{\left|C \backslash C_{i}\right|} \frac{1}{\left|C \backslash C_{j}\right|} \geqslant \frac{3 k-\left|C_{i}\right|-\left|C_{j}\right|}{\left(3 k-\left|C_{i}\right|\right)\left(3 k-\left|C_{j}\right|\right)}$. 
Let $f(x, y)=\frac{3 k-x-y}{(3 k-x)(3 k-y)}$ for $x, y \in[0, k] \subset \mathbb{R}$. Since $\frac{\partial f(x, y)}{\partial x}, \frac{\partial f(x, y)}{\partial y} \leqslant 0$ for all $x, y \in$ $[0, k]$, we infer that for any pair $x, y \in[0, k], f(x, y) \geqslant f(x, k) \geqslant f(k, k)=1 / 4 k$. Hence $p \geqslant 1 / 4 k$.

So the probability that $v_{i}$ and $v_{j}$ get different colors in a particular coloring $\leqslant\left(1-\frac{1}{4 k}\right)$ and the probability that $v_{i}$ and $v_{j}$ get different colors in all the $r$ colorings $\leqslant\left(1-\frac{1}{4 k}\right)^{r} \leqslant$ $e^{\frac{-r}{4 k}}$. Hence, the probability that some pair of non-adjacent vertices get different colors in all the $r$ colorings $<n^{2} e^{\frac{-r}{4 k}}$. If $r \geqslant 8 k \ln n=5.545 k \log n, n^{2} e^{\frac{-r}{4 k}} \leqslant 1$. Thus, if $r=\lceil 5.545 k \log n\rceil$, then every pair of non-adjacent vertices in the graph gets the same color in at least one of the $r$ colorings described above. To ensure that no pair of vertices get the same color in all colorings we also consider an $(r+1)$-th coloring where all vertices get a unique color. Thus $\operatorname{pdim}(G) \leqslant\lceil 5.545 k \log n\rceil+1$.

\section{References}

[1] P. Alles. Dimension of amalgamated graphs and trees. Czechoslovak Mathematical Journal, 36(3):393-416, 1986.

[2] N. Alon. Covering graphs by the minimum number of equivalence relations. Combinatorica, 6(3):201-206, 1986.

[3] R.C. Bose, S.S. Shrikhande, and E.T. Parker. Further results on the construction of mutually orthogonal Latin squares and the falsity of Euler's conjecture. Canad. J. Math, 12:189-203, 1960.

[4] L.S. Chandran and N. Sivadasan. Boxicity and treewidth. Journal of Combinatorial Theory, Series B, 97(5):733-744, 2007.

[5] Jeffery R. Cooper. Product dimension of a random graph. Masters thesis, Miami University, Oxford, Ohio, 2010.

[6] N. Eaton and V. Rödl. Graphs of small dimensions. Combinatorica, 16(1):59-85, 1996.

[7] P. Erdös and A.B. Evans. Representations of graphs and orthogonal Latin square graphs. Journal of Graph Theory, 13(5):593-595, 1989.

[8] A.B. Evans. Representations of disjoint unions of complete graphs. Discrete mathematics, 307(9):1191-1198, 2007.

[9] A.B. Evans, G. Isaak, and D.A. Narayan. Representations of graphs modulo n. Discrete Mathematics, 223(1):109-123, 2000.

[10] Z. Füredi. On the Prague dimension of Kneser graphs. Numbers, information, and complexity, page 125, 2000.

[11] Ida Kantor. Graphs, codes, and colorings. Ph.D thesis, Graduate College of the University of Illinois at Urbana-Champaign, 2010.

[12] A.M.C.A. Koster, H.L. Bodlaender, and S.P.M. Van Hoesel. Treewidth: computational experiments. Research Memoranda 0001, Maastricht : METEOR, Maastricht Research School of Economics of Technology and Organization, 2001. 
[13] L. Lovász, J. Nešetřil, and A. Pultr. On a product dimension of graphs. Journal of Combinatorial Theory, Series B, 29(1):47-67, 1980.

[14] C. Lu. A note on lower bounds of treewidth for graphs. In International Mathematical Forum, volume 2, pages 2893-2898, 2007.

[15] J. Nešetřil and V. Rödl. A simple proof of the Galvin-Ramsey property of the class of all finite graphs and a dimension of a graph. Discrete Mathematics, 23(1):49-55, 1978.

[16] S. Poljak and A. Pultr. On the dimension of trees. Discrete Mathematics, 34(2):165$171,1981$. 